\title{
Ação de inseticidas naturais no controle de Spodoptera frugiperda (J.E. Smith, 1797) (Lepidoptera: Noctuidae) em milho cultivado em agroecossistema de várzea
}

\author{
Action of natural insecticide in control of Spodoptera frugiperda (J.E. Smith, 1797) (Lepidoptera: \\ Noctuidae) in corn crop cultivated in low agroecossistem
}

\author{
José Farid Maia Lima I Anderson Dionei Grützmacher ${ }^{I^{*}}$ Uemerson Silva da Cunha" \\ Marilda Pereira Porto ${ }^{\mathrm{III}}$ José Francisco da Silva Martins ${ }^{\mathrm{III}}$ Gabriel Olle Dalmazo ${ }^{\mathrm{II}}$
}

RESUMO

Estudou-se a eficiência de inseticidas naturais no controle de Spodoptera frugiperda (J.E. Smith, 1797) (Lepidoptera: Noctuidae), na cultura do milho em agroecossistema de várzea, sob infestação natural. O experimento foi conduzido a campo na área experimental da Embrapa Clima Temperado - Estação Experimental Terras Baixas, no ano agrícola 2005/2006. O delineamento experimental foi de blocos ao acaso em esquema fatorial (9x2), com 9 tratamentos: T1- NeemAzal-T/S (0,25\%); T2- NeemAzalT/S (0,5\%); T3- Rotenat CE (0,5\%); T4- Natuneem agrícola (0,5\%); T5- Rotenat CE + Natuneem agrícola $(0,25+0,25 \%)$; T6- Dalneem emulsionável (0,5\%); T7- Organic neem $(0,5 \%)$ T8- Match CE (0,1\%) (padrão) e T9- Água (testemunha) com duas épocas de semeadura e quatro repetições. Realizaram-se três aplicações dos tratamentos por época, determinando-se a porcentagem de plantas atacadas oito dias após cada aplicação e o rendimento de grãos em $\mathrm{kg} \mathrm{ha}^{-1}$. Obteve-se diferença significativa no controle da lagarta-do-cartucho entre épocas de semeadura com maior eficiência de controle para a primeira época, destacando-se dentre os inseticidas naturais o NeemAzal-T/S (0,5\%) e o Dalneem emulsionável (0,5\%), com grande potencial de uso em semeaduras realizadas no mês de novembro. $O$ rendimento de grãos entre épocas não diferiu estatisticamente, destacando-se dentre os inseticidas naturais o NeemAzal - T/S a 0,5\%, tornando-se uma alternativa no controle da S. frugiperda. Há uma correlação negativa e significativa entre plantas atacadas e rendimento de grãos em $\mathrm{kg} \mathrm{ha}^{-1}$, com maior potencial de controle para a primeira época de semeadura.

Palavras-chave: lagarta-do-cartucho, Zea mays, controle, Azadirachta indica.

\section{ABSTRACT}

The efficiency of natural insecticide was studied in control Spodoptera frugiperda (J.E. Smith, 1797) (Lepidoptera: Noctuidae) in corn crop cultivated in low agroecossistem, under natural infestation. The experiment was conducted at the experimental area of Embrapa Clima Temperado - Estação Experimental Terras Baixas, agricultural year 2005/2006. The experimental diagram was of blocks in factorial design $(9 \times 2)$, with 9 treatments: T1- NeemAzal-T/S (0.25\%); T2- NeemAzalT/S (0.5\%); T3- Rotenat CE (0.5\%); T4- Natuneem agrícola (0.5\%); T5- Rotenat CE + Natuneem agrícola $(0.25+0.25 \%)$; T6- Dalneem emulsionable (0.5\%); T7- Organic neem (0.5\%); T8- Match CE (0.1\%) (Standard) and T9- Water (check), with two times of sowing and four repetitions. Three applications of treatments were carried for time, determining the percentage of plants attacked eight days after each application and yield of grain in $\mathrm{kg} \mathrm{ha}^{-1}$. There was significant difference in control of fall armyworm among time of sowing, with a major efficiency of control for first time, pointing out among the natural insecticide the NeemAzal - T/S (0.5\%) and Dalneem emulsionable $(0.5 \%)$, with great potential of use in sowing carried out in November. The yield doesn't differ among time, pointing out among the natural insecticide the Neem Azal - T/ $S$ at $0.5 \%$, bringing an alternative in control of $S$. frugiperda. There is a negative and significant correlation among plants attacked and yield of grain in $\mathrm{kg} \mathrm{ha}^{-1}$, with major potential of control for the first time sowing.

Key words: Fall armyworm, Zea mays, control, Azadirachta indica.

'Universidad Amazónica de Pando (UAP), Pando, Bolívia. Programa de Pós-graduação em Fitossanidade, Faculdade de Agronomia Eliseu Maciel (FAEM), Universidade Federal de Pelotas (UFPel), Pelotas, RS, Brasil.

I"Departamento de Fitossanidade, FAEM, UFPel, CP 354, 96010-900, Pelotas, RS, Brasil. E-mail: adgrutzm@ufpel.tche.br.

*Autor para correspondência.

IIIEmbrapa Clima Temperado, Pelotas, RS, Brasil. 


\section{INTRODUÇÃO}

Na região Sul do Estado do Rio Grande do Sul, em solos de várzea, onde o cultivo do arroz irrigado se dá em grandes áreas, a cultura do milho tem assumido grande importância como alternativa no sistema de rotação de culturas, auxiliando principalmente no controle de plantas daninhas como o arroz vermelho (PORTO et al., 1998). Nesse contexto, segundo GRÜTZMACHER et al. (2000a), como as culturas de arroz irrigado e sorgo são hospedeiras da lagarta-do-cartucho, a proximidade das áreas cultivadas com estas gramíneas pode intensificar o ataque do inseto aos milharais.

A lagarta-do-cartucho, Spodoptera frugiperda (J.E. Smith, 1797) (Lepidoptera: Noctuidae), é considerada a mais importante praga do milho no Brasil (CRUZ \& MONTEIRO, 2004), ocasionando perdas na produção de até 38,7\% (WILLIAMS \& DAVIS, 1990; CRUZ et al., 1996). Ela ataca preferencialmente o “cartucho” da planta, consumindo grande parte das folhas antes destas abrirem. Quando o ataque ocorre nos primeiros estádios da cultura, ele pode provocar a morte das plantas, reduzindo a população inicial. Em estádios mais adiantados, podese encontrar a lagarta atacando o pendão e até mesmo as espigas em formação (WAQUIL et al., 1982), ocorrendo em todas as fases de desenvolvimento da planta de milho e em todas as suas estruturas aéreas (BUNTIN, 1986). Tais características de ocorrência se devem também à alta mobilidade da espécie e a sua capacidade para colonizar rapidamente os cultivos de milho quando as condições são favoráveis para seu normal desenvolvimento e sobrevivência (MURUA \& VIRLA, 2004).

O controle da lagarta-do-cartucho geralmente é realizado com produtos químicos sintéticos. As aplicações de inseticidas freqüentemente são tardias e acima do nível de controle (20\% no período vegetativo e $10 \%$ no período reprodutivo) GRÜTZMACHER et al. (2000b), os quais nem sempre são eficientes, podendo acarretar diversos problemas, tais como: resíduos em alimentos, eliminação de inimigos naturais, intoxicação dos aplicadores, seleção de populações de pragas resistentes aos inseticidas, entre outros efeitos diretos e indiretos (DIEZ - RODRIGUEZ \& OMOTO, 2001). A busca de outros métodos de controle inclui a utilização de produtos menos agressivos ao ambiente, como, por exemplo, inseticidas de origem vegetal (VENDRAMIM, 1997).

Produtos naturais, provenientes de plantas, podem ser uma alternativa ao manejo da $\boldsymbol{S}$. frugiperda. Nesse contexto insere-se a utilização de extratos de plantas popularmente conhecidos como inseticidas naturais (GUERRA, 1985; BOGORNI \& VENDRAMIM, 2003).

A Azadirachta indica A. Juss., da família Meliaceae, popularmente denominada nim, tem sido mundialmente estudada quanto ao seu potencial inseticida, de maneira que seus extratos têm se revelado tão potentes quanto alguns inseticidas comerciais, principalmente em relação aos lepidópteros, que são os mais sensíveis às substâncias derivadas do nim (SCHMUTTERER, 1990). Esse efeito tem sido particularmente demonstrado em condições de laboratório para várias espécies (SIMMONDS, 2000). Segundo MARTINEZ (2002), o nim é conhecido há 5.000 anos e apresenta ação contra mais de 430 espécies de pragas que ocorrem em diversos países, causando múltiplos efeitos, tais como: repelência, interrupção do desenvolvimento e da ecdise, atraso no desenvolvimento, redução na fertilidade e fecundidade, e várias outras alterações no comportamento e na fisiologia dos insetos que podem levá-los à morte. O nim apresenta uma série de compostos limonóides, dentre os quais a azadiractina é o que ocorre em maior concentração e que apresenta maior atividade tóxica contra insetos. É utilizada principalmente na forma de óleo ou na forma de extratos aquosos ou orgânicos. As vantagens do nim em relação a outras plantas inseticidas são a atividade sistêmica, a eficiência em baixas concentrações, a baixa toxicidade a mamíferos e a menor probabilidade de desenvolvimento de resistência pela ocorrência de um complexo de princípios ativos (VENDRAMIM \& CASTIGLIONI, 2000).

Trabalhos de controle da $\boldsymbol{S}$. frugiperda em milho com inseticidas naturais a campo são inexistentes no Brasil. Em Cuba, PÉREZ et al. (1997) avaliaram três inseticidas naturais derivados do nim no controle da lagarta-do-cartucho do milho a campo, destacando a eficiência dos mesmos com 3 a 4 aplicações, sem, no entanto, conseguir superar o inseticida padrão comercial.

Esta pesquisa teve como objetivo avaliar a eficiência de inseticidas naturais à base de nim e rotenona no controle de $\boldsymbol{S}$. frugiperda, na cultura do milho em agroecossistema de várzea, em duas épocas de semeadura, sob infestação natural a campo.

\section{MATERIAL E MÉTODOS}

O experimento foi conduzido a campo na Estação Experimental de Terras Baixas (ETB) do Centro de Pesquisa Agropecuária de Clima Temperado (CPACT) da Empresa Brasileira de Pesquisa

Ciência Rural, v.38, n.3, mai-jun, 2008. 
Agropecuária (Embrapa), localizada no município do Capão do Leão, Rio Grande do Sul (latitude: 3149'12,2” Sul, longitude: $52^{\circ} 27^{\prime} 38,8^{\prime \prime}$ Oeste e altitude aproximada de $22 \mathrm{~m}$ ), durante o ano agrícola 2005/2006, sob infestação natural de $\boldsymbol{S}$. frugiperda.

Realizou-se a semeadura da cultivar de milho "Pioneer 30F33” (ciclo precoce), no espaçamento de $0,7 \mathrm{~m}$ entre linhas e quatro plantas por metro linear. As parcelas foram compostas de oito fileiras com $5 \mathrm{~m}$ de comprimento, resultando em uma área total de $28 \mathrm{~m}^{2}$, sendo considerada como área útil $16,8 \mathrm{~m}^{2}$, considerandose as seis fileiras centrais e desprezando-se meio metro em cada extremidade.

O delineamento experimental utilizado foi de blocos ao acaso, em esquema fatorial (9x2), com nove tratamentos (Tabela 1) e duas épocas de semeadura: I época (09 de novembro/05) e II época (08 de dezembro/ $05)$, cada uma com quatro repetições. Em cada época de semeadura, realizaram-se três aplicações dos tratamentos: I época (8/12/05, 27/12/05 e 6/01/06) e II época (05/01/06, 23/01/06 e 06/02/06), utilizando-se pulverizador costal a $\mathrm{CO}_{2}$ com bico tipo leque XR 110:02 e volume de calda de 300 $\mathrm{L} \mathrm{ha}^{-1}$ com jato dirigido ao cartucho.

As avaliações foram realizadas no oitavo dia, após cada pulverização, abrindo-se então os cartuchos de 10 plantas coletadas ao acaso na área útil de cada parcela e contando-se o número de plantas atacadas e com lagartas vivas. A colheita foi realizada por ocasião da maturação fisiológica dos grãos, sendo determinado o rendimento em $\mathrm{kg} \mathrm{ha}^{-1}$.

Para a análise estatística, utilizou-se o programa R (R DEVELOPMENT CORE TEAM, 2006), sendo que os dados de plantas atacadas e o rendimento de grãos em $\mathrm{kg} \mathrm{ha}^{-1}$ foram submetidos à análise da variância (ANOVA). Quando o teste F da ANOVA indicou significância de 5\% de probabilidade de erro, procederam-se as análises complementares através do teste de Tukey a 5\% de probabilidade. Para determinar a eficiência de controle, empregou-se a fórmula de Abbott (ABBOTT, 1925). Determinaram-se as análises de regressão linear entre plantas atacadas com lagartas vivas no cartucho (\%) e rendimento de grãos em $\mathrm{kg} \mathrm{ha}^{-1}$, sendo a significância determinada pelo teste de F a 5\% de probabilidade.

\section{RESULTADOS E DISCUSSÃO}

Na avaliação dos inseticidas naturais por época e aplicações (Tabela 2), constatou-se, na primeira época de semeadura, diminuição do percentual de plantas atacadas com lagartas vivas de $\boldsymbol{S}$. frugiperda no cartucho após cada pulverização, sendo menor após a terceira, que diferiu significativamente das demais para a maioria dos tratamentos. Para a primeira época de semeadura (Tabela 2), na primeira e na segunda aplicação, todos os inseticidas, à exceção do Match CE (0,1\%), não diferiram da testemunha, com destaque na primeira aplicação para Rotenat CE (0,5\%), NeemAzal-T/S (0,5\%), NeemAzal-T/S (0,25\%), Rotenat $\mathrm{CE}+$ Natuneem $(0,25 \%+0,25 \%)$ e Dalneem emulsionável(0,5\%), os quais não diferiram do tratamento padrão (Match CE). Para a segunda aplicação, destacaram-se os inseticidas naturais NeemAzal-T/S (0,5\%), Dalneem emulsionável (0,5\%) e Organic neem $(0,5 \%)$, não diferindo do tratamento padrão. No entanto, na terceira aplicação, o NeemAzalT/S (0,5\%) e o Dalneem emulsionável (0,5\%) diferiram da testemunha significativamente, porém reduziram o número de plantas atacadas de forma semelhante ao tratamento padrão.

Tabela 1 - Inseticidas avaliados no controle da $\boldsymbol{S}$. frugiperda, em milho cultivado em várzea, sob infestação natural em duas épocas de semeaduras (09 de novembro e 08 de dezembro/2005). Capão do Leão - RS, ano agrícola 2005/2006.

\begin{tabular}{|c|c|c|c|c|}
\hline Produto & Nome comercial & Concentração i.a. (\%) & Concentração p.c. (\%) ${ }^{1}$ & Empresa fabricante \\
\hline Azadirachtina A & NeemAzal-T/S & 1 & 0,25 & Trifolio - M \\
\hline Azadirachtina A & NeemAzal-T/S & 1 & 0,50 & Trifolio - M \\
\hline Extrato vegetal (Derris sp.) & Rotenat CE & 5 & 0,50 & Natural Rural \\
\hline Óleo de nim (Azadirachta indica) & Natuneem agrícola & n.i. & 0,50 & Natural Rural \\
\hline Oleo de nim + Extrato vegetal & Rotenat CE+ Natuneem & n.i. & $0,25+0,25$ & Natural Rural \\
\hline Extrato vegetal (Azadirachta indica) & Dalneem emulsionável & n.i. & 0,50 & Dalquim \\
\hline Óleo de nim (Azadirachta indica) & Organic neem & n.i. & 0,50 & Dalquim \\
\hline Lufenurom & Match CE (padrão) & 5 & 0,10 & Syngenta \\
\hline Testemunha (água) & --- & ---- & --- & ---- \\
\hline
\end{tabular}

${ }^{\text {n.i. }}$ Concentração não informada pela empresa fabricante.

${ }^{1}$ Volume de calda utilizado a campo (300 L.ha $\left.{ }^{-1}\right)$. 
Tabela 2 - Efeito de inseticidas naturais sobre plantas atacadas com lagartas vivas de $\boldsymbol{S}$. frugiperda no cartucho (\%), em duas épocas de semeadura e com três aplicações por época. Capão do Leão - RS, ano agrícola 2005/2006.

\begin{tabular}{|c|c|c|c|c|c|c|}
\hline \multicolumn{7}{|c|}{ Primeira época de semeadura (09/11) } \\
\hline \multirow{2}{*}{ Tratamento pc (\%) } & \multicolumn{2}{|c|}{---I Aplicação 19 DAE ${ }^{1}$--- } & \multicolumn{2}{|c|}{----II Aplicação 38 DAE---- } & \multicolumn{2}{|c|}{----III Aplicação 48 DAE---- } \\
\hline & P. atacadas ${ }^{2}$ & $\mathrm{EC}^{3}$ & P. atacadas & EC & P. atacadas & EC \\
\hline NeemAzal - T/S $(0,25)$ & $35,0 \mathrm{ab} A$ & 22,2 & 32,5 a $\mathrm{A}$ & 13,3 & $10,0 \mathrm{ab} B$ & 69,3 \\
\hline NeemAzal - T/S $(0,5)$ & $27,5 \mathrm{ab} A$ & 38,9 & 22,5 ab A & 40,0 & 2,5 b B & 92,3 \\
\hline Rotenat CE $(0,5)$ & $22,5 \mathrm{ab} A$ & 50,0 & 32,5 a $A$ & 13,3 & 17,5 ab A & 46,2 \\
\hline Natuneem agrícola $(0,5)$ & 40,0 a $\mathrm{A}$ & 11,1 & 35,0 a $\mathrm{A}$ & 6,7 & 17,5 ab B & 46,2 \\
\hline Rotenat CE + Natuneem $(0,25+0,25)$ & $32,5 \mathrm{ab} A$ & 27,8 & 35,0 a $\mathrm{A}$ & 6,7 & 22,5 ab A & 30,8 \\
\hline Dalneem emulsionável $(0,5)$ & $35,0 \mathrm{ab} A$ & 22,2 & 22,5 ab A & 40,0 & $2,5 \mathrm{~b} \mathrm{~B}$ & 92,3 \\
\hline Organic neem $(0,5)$ & 37,5 a $\mathrm{A}$ & 16,7 & 30,0 ab A & 20,0 & 7,5 ab B & 76,9 \\
\hline Match CE $(0,1)$ (padrão) & 10,0 b A & 77,8 & 10,0 b A & 73,3 & $7,5 \mathrm{ab} \mathrm{A}$ & 76,9 \\
\hline Testemunha (água) & 45,0 a $\mathrm{A}$ & - & 37,5 a $\mathrm{A}$ & - & 32,5 a $\mathrm{A}$ & - \\
\hline Média geral & $31,7 \mathrm{~A}$ & & $28,6 \mathrm{~A}$ & & $13,3 \mathrm{~B}$ & \\
\hline $\mathrm{CV} \%$ & 39,4 & & 32,8 & & 89,0 & \\
\hline \multicolumn{7}{|c|}{ Segunda época de semeadura (08/12) } \\
\hline \multirow{2}{*}{ Tratamento p.c. (\%) } & \multicolumn{2}{|c|}{----I Aplicação 20 DAE---- } & \multicolumn{2}{|c|}{----II Aplicação 38 DAE---- } & \multicolumn{2}{|c|}{----III Aplicação 52 DAE---- } \\
\hline & P. atacadas & EC & P. atacadas & EC & P. atacadas & EC \\
\hline NeemAzal - T/S $(0,25)$ & 57,5 a $\mathrm{AB}$ & 20,7 & 32,5 ab B & 45,8 & 72,5 a $\mathrm{A}$ & 3,3 \\
\hline NeemAzal - T/S $(0,5)$ & 55,0 a $A$ & 24,1 & 45,0 ab A & 25,0 & 57,5 a $A$ & 23,3 \\
\hline Rotenat CE $(0,5)$ & 60,0 a $\mathrm{A}$ & 17,2 & 57,5 a $\mathrm{A}$ & 4,2 & 75,0 a $\mathrm{A}$ & 0,0 \\
\hline Natuneem agrícola $(0,5)$ & 60,0 a $\mathrm{A}$ & 17,2 & 50,0 a $\mathrm{A}$ & 16,7 & 72,5 a $\mathrm{A}$ & 3,3 \\
\hline Rotenat CE + Natuneem $(0,25+0,25)$ & 65,0 a $\mathrm{A}$ & 10,3 & $45,0 \mathrm{ab} A$ & 25,0 & 67,5 а $\mathrm{A}$ & 10,0 \\
\hline Dalneem emulsionável $(0,5)$ & 57,5 а $\mathrm{AB}$ & 20,7 & 32,5 ab B & 45,8 & 60,0 a $\mathrm{A}$ & 20,0 \\
\hline Organic neem $(0,5)$ & 67,5 a $A$ & 6,9 & 35,0 ab B & 41,7 & 60,0 a $\mathrm{AB}$ & 20,0 \\
\hline Match CE $(0,1)$ (padrão) & 45,0 a $\mathrm{A}$ & 37,9 & 7,5 b B & 87,5 & 42,5 a $\mathrm{A}$ & 43,3 \\
\hline Testemunha (água) & 72,5 a $\mathrm{A}$ & - & 60,0 a $\mathrm{A}$ & - & 75,0 a $\mathrm{A}$ & - \\
\hline Média geral & $60,0 \mathrm{~A}$ & & $40,6 \mathrm{~B}$ & & $64,7 \mathrm{~A}$ & \\
\hline $\mathrm{CV} \%$ & 26,5 & & 41,4 & & 23,37 & \\
\hline
\end{tabular}

${ }^{1}$ Dias após a emergência das plantas de milho (DAE).

${ }^{2}$ Médias seguidas por letras distintas, minúscula na coluna e maiúscula na linha, diferem significativamente entre si pelo teste de Tukey em nível de 5\% de significância.

${ }^{3}$ Eficiência de controle em porcentagem, calculada pela fórmula de Abbott (ABBOTT, 1925).

Na segunda época de semeadura (Tabela 2), houve diferenças significativas entre aplicações, cabendo destacar que, na primeira aplicação, todos os inseticidas, inclusive o tratamento padrão, igualaramse à testemunha em função da elevada infestação inicial (Tabela 3), a qual foi de $90 \%$ de plantas atacadas, superando significativamente a infestação de $40 \%$ de plantas atacadas da primeira época, sendo o dobro do nível de controle estabelecido para o período vegetativo (GRÜTZMACHER et al., 2000b). Estes resultados estão de acordo com os obtidos por PÉREZ et al. (1997), os quais observaram eficiência similar para três produtos naturais à base de nim (Cubanim-t, Oleonim-80 CE e Oleonim-50 CE) no controle da lagarta-do-cartucho, decorrente de três e quatro aplicações, sem, no entanto, superar o tratamento padrão (Karate). SOSA (2002) observou crescimento populacional de $\boldsymbol{S}$. frugiperda nos meses de novembro e dezembro, chegando ao ponto máximo em janeiro, concluindo que as semeaduras tardias estão mais expostas à presença da praga. Além do mais, segundo GRÜTZMACHER et al. (2000a), a proximidade de áreas cultivadas com arroz e sorgo intensifica o ataque do inseto aos milharais, já que ambas as espécies são hospedeiras alternativas da lagarta-do-cartucho.

Dentre os inseticidas naturais avaliados (Tabela 3), observou-se diferença significativa em relação a plantas atacadas, diferindo significativamente entre épocas de semeadura, com destaque na primeira época para NeemAzal-T/S (0,5\%) e Dalneem

Ciência Rural, v.38, n.3, mai-jun, 2008. 
Tabela 3 - Efeito de inseticidas naturais sobre plantas atacadas com lagartas vivas de $\boldsymbol{S}$. frugiperda no cartucho (\%) e eficiência de controle em milho cultivado em várzea, em duas épocas de semeadura (09/11/05 e 08/12/05). Capão do Leão - RS, ano agrícola $2005 / 2006$.

\begin{tabular}{|c|c|c|c|c|}
\hline \multirow{2}{*}{ Tratamento p.c. (\%) } & \multicolumn{2}{|c|}{----------I época (09/11)---------- } & \multicolumn{2}{|c|}{----------II época (08/12)-------- } \\
\hline & Plantas atacadas ${ }^{1}$ & $\mathrm{EC}^{2}$ & Plantas atacadas & EC \\
\hline NeemAzal - T/S $(0,25)$ & 25,83 ab B & 32,6 & 54,17 ab A & 19,8 \\
\hline NeemAzal - T/S $(0,5)$ & 17,50 bc B & 54,3 & $50,00 \mathrm{~b} \mathrm{~A}$ & 25,9 \\
\hline Rotenat CE $(0,5)$ & 24,16 ab B & 36,0 & $62,50 \mathrm{ab} A$ & 7,4 \\
\hline Natuneem agrícola $(0,5)$ & 30,83 ab B & 19,6 & 59,17 ab A & 12,3 \\
\hline Rotenat CE + Natuneem $(0,25+0,25)$ & 29,17 ab B & 23,9 & $57,50 \mathrm{ab} A$ & 14,8 \\
\hline Dalneem emulsionável $(0,5)$ & 20,00 bc B & 45,9 & $50,00 \mathrm{~b} A$ & 25,9 \\
\hline Organic neem $(0,5)$ & $25,00 \mathrm{ab} B$ & 34,8 & 54,17 ab A & 19,8 \\
\hline Match CE $(0,1)$ (padrão) & 9,17 с B & 76,1 & 31,67 с A & 53,1 \\
\hline Testemunha (água) & 38,33 a B & - & 67,50 a $A$ & - \\
\hline Média geral & $24,44 \mathrm{~B}$ & & $54,07 \mathrm{~A}$ & \\
\hline $\mathrm{CV} \%$ & 25,88 & & 11,70 & \\
\hline Infestação Inicial (\%) & $40 \mathrm{~B}$ & & $90 \mathrm{~A}$ & \\
\hline
\end{tabular}

${ }^{1}$ Médias seguidas por letras distintas, minúsculas na coluna e maiúsculas na linha, diferem significativamente entre si pelo teste de Tukey em nível de 5\% de significância.

${ }^{2}$ Eficiência de controle em porcentagem, calculada pela fórmula de Abbott (ABBOTT, 1925).

emulsionável (0,5\%), os quais, além de equivalerem-se ao inseticida padrão (Match CE a 0,1\%), diferiram significativamente da testemunha. Na segunda época, de maneira similar à primeira, destacaram-se dentre os inseticidas naturais NeemAzal-T/S (0,5\%) e Dalneem emulsionável $(0,5 \%)$, os quais, embora não tenham superado o tratamento padrão, reduziram significativamente o percentual de plantas atacadas em relação à testemunha, com menor eficiência de controle comparada à primeira época de semeadura (variando de 43 a 53\%), que foi provavelmente causada pela baixa eficiência de controle na segunda época de 25,9\%. SILVA (1999) demonstrou que a eficiência de inseticidas no controle de $\boldsymbol{S}$. frugiperda varia em função da época, sendo menor em épocas tardias, constatando que o insucesso no controle da praga deve-se à realização do controle em forma tardia, mesmo que seja realizado quando existem altas infestações e presença de lagartas grandes.

Para a variável rendimento de grãos em kg $\mathrm{ha}^{-1}$, não houve interação entre os inseticidas testados e as épocas de semeadura, não se constatando diferença significativa entre épocas (Tabela 4). No entanto, tanto na primeira como na segunda época de semeadura, dentre os inseticidas naturais avaliados, destacou-se o NeemAzal-T/S (0,5\%), o qual diferiu significativamente da testemunha equivalendo-se ao inseticida padrão (Match CE a 0,1\%) (Tabela 4). Estes resultados são corroborados pela eficiência de controle da lagarta-do-cartucho obtida na primeira época, que se deve à menor infestação inicial (Tabela 3), tornando o NeemAzal-T/S (0,5\%) uma alternativa a mais no controle da praga, em semeaduras realizadas no mês de novembro.

Além disso, é observada a existência de uma correlação negativa e significativa entre plantas atacadas (\%) e rendimento de grãos $\left(\mathrm{kg} \mathrm{ha}^{-1}\right)$, de modo que a cada $1 \%$ de plantas atacadas o rendimento de grãos decresce $97,19 \mathrm{~kg} \mathrm{ha}^{-1}$ e 76,36kg ha-1 na primeira e na segunda época (Figura 1) respectivamente. Destacase o ajuste proporcionado pelo modelo matemático, por meio do qual pode-se inferir que o potencial produtivo da segunda época é superior ao da primeira em 1.501,13kg ha-1 (quando a \% de plantas atacadas for igual a zero) mesmo que associado à alta infestação inicial, fazendo com que se tenha uma menor eficiência no controle na segunda época (dezembro), não diferindo o rendimento dos grãos entre épocas de semeadura. Resultados que dão crédito às recomendações feitas por VENDRAMIM (1997), que sugere a utilização de inseticidas naturais quando existam baixas densidades populacionais da praga, já que o controle deve ser feito mais preventivamente do que como curativo.

Em virtude da alta infestação inicial da lagarta-do-cartucho $S$. frugiperda na cultura do milho em agroecossistema de várzea, é necessário rever os 
Tabela 4 - Rendimento de grãos em kg ha ${ }^{-1}$ para milho cultivado em várzea, em duas épocas de semeadura (09/11/05 e 08/12/05), após a aplicação dos tratamentos para o controle de $\boldsymbol{S}$. frugiperda com inseticidas naturais em condições de campo. Capão do Leão - RS, ano agrícola 2005/2006.

\begin{tabular}{lcc}
\hline & & \\
Tratamento p.c. (\%) & I época (08/12) \\
\hline NeemAzal - T/S (0,25) & I época (09/11) \\
NeemAzal - T/S (0,5) & 4745,7 ab A & 4973,0 abc A \\
Rotenat CE (0,5) & 6502,5 a A & 5948,7 ab A \\
Natuneem agrícola (0,5) & 4833,2 ab A & 4406,7 abc A \\
Rotenat CE + Natuneem (0,25+0,25) & 4110,0 bc A \\
Dalneem emulsionável (0,5) & 4143,7 b A & 4839,0 abc A \\
Organic neem (0,5) & 3990,7 b A & 4536,5 abc A \\
Match CE (0,1) (padrão) & 5340,7 ab A & 4665,5 abc A \\
Testemunha (água) & 5894,7 ab A & 6496,5 a A \\
\hline Média geral & 6753,7 a A & 3710,0 c A \\
CV\% & 4156,0 b A & 19,8 \\
\hline
\end{tabular}

${ }^{1}$ Médias seguidas por letras distintas, minúsculas na coluna e maiúsculas na linha, diferem significativamente entre si pelo teste de Tukey em nível de 5\% de significância.

atuais níveis de controle (GRÜTZMACHER et al., 2000b), bem como realizar um estudo da viabilidade econômica das aplicações seqüenciais (acumuladas) dos inseticidas naturais (no mínimo três aplicações), sendo que as mesmas devem complementar-se com testes de seletividade a inimigos naturais das pragas do milho.

\section{CONCLUSÕES}

Há diferença significativa no controle da lagarta-do-cartucho $\boldsymbol{S}$. frugiperda entre épocas de semeadura, com uma maior eficiência de controle para a primeira época, destacando-se dentre os inseticidas naturais o NeemAzal-T/S (0,5\%) e o Dalneem emulsionável (0,5\%).

O rendimento de grãos não difere entre épocas, destacando-se dentre os inseticidas naturais o NeemAzal-T/S a 0,5\%, tornando-se uma alternativa no controle da $\boldsymbol{S}$. frugiperda em agroecossistema de várzea. Há uma correlação negativa e significativa entre plantas atacadas e rendimento de grãos em kg.ha ${ }^{-1}$, com maior potencial de controle da lagarta-do-cartucho do milho para a primeira época de semeadura, realizada no mês de novembro.

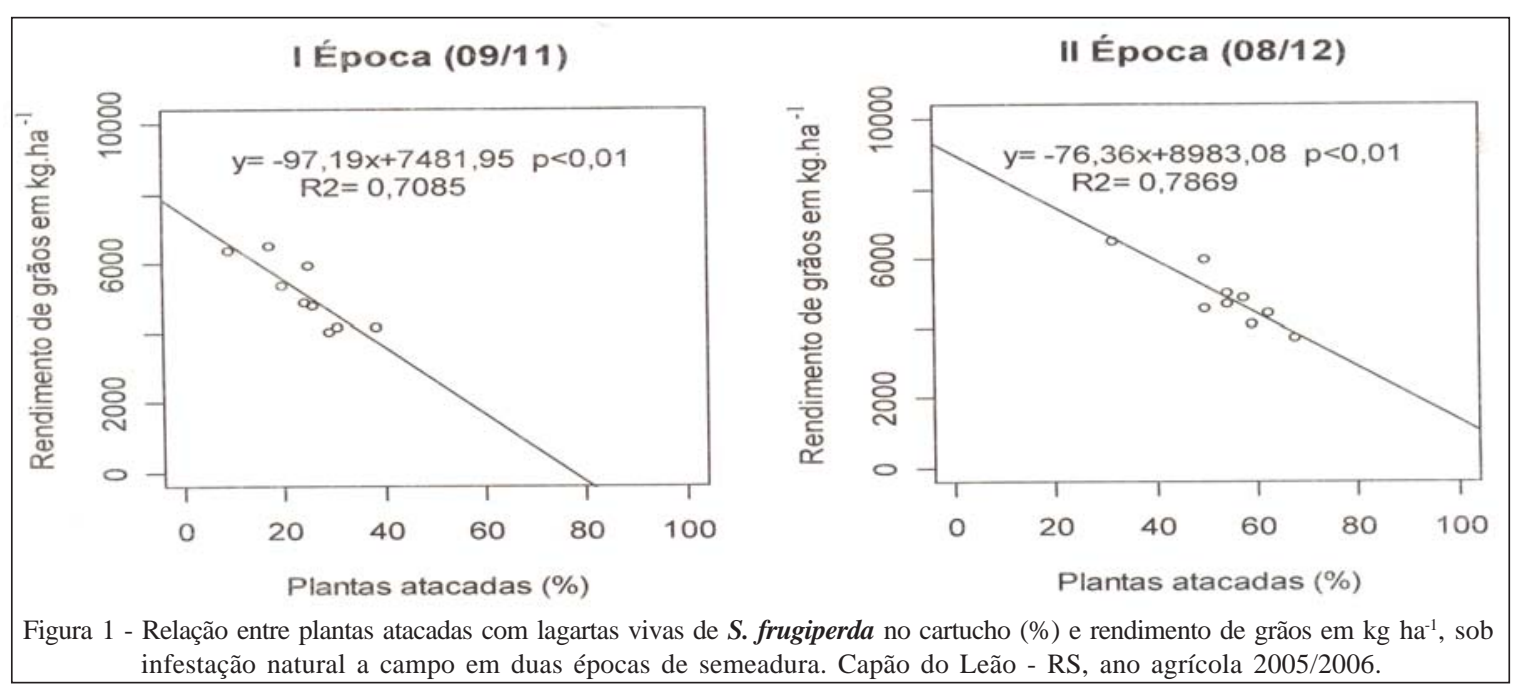

Ciência Rural, v.38, n.3, mai-jun, 2008. 


\section{REFERÊNCIAS}

ABBOTT, W.S. A method of computing the effectiveness of an insecticide. Journal of Economic Entomology, Lanham, v.18, p.265-267, 1925.

BOGORNI, P.C.; VENDRAMIM J.D. Bioatividade de extratos aquosos de Trichilia spp. sobre Spodoptera frugiperda (J.E. Smith) (Lepidoptera: Noctuidae) em milho. Neotropical Entomology, Londrina, v.32, p.665-669, 2003.

BUNTIN, G.D. A review of plant response to fall armyworm, Spodoptera frugiperda (J.E. Smith), injury to select field and forage crops. Florida Entomologist, Gainesville, v.69, n.3, p.549-559, 1986

CRUZ, I.; MONTEIRO, M.A.R. Controle biológico da lagarta do cartucho do milho Spodoptera frugiperda utilizando o parasitóide de ovos Trichogramma pretiosum. Sete Lagoas: Embrapa Milho e Sorgo, 2004. 4p. (Comunicado Técnico).

CRUZ, I. et al. Efeito do nível de saturação de alumínio em solo ácido sobre os danos de Spodoptera frugiperda (J. E. Smith) em milho. Anais da Sociedade Entomológica do Brasil, Londrina, v.25, p.293-297, 1996.

DIEZ-RODRIGUEZ, G.I.; OMOTO, C. Herança da resistência de Spodoptera frugiperda (J.E. Smith) (Lepidoptera: Noctuidae) a lambda-cialotrina. Neotropical Entomology, Londrina, v.30, p.311-316, 2001.

GUERRA, M.S. Receituário caseiro: alternativas para o controle de pragas e doenças de plantas cultivadas e de seus produtos. Brasília: Embrater, 1985 . 166p.

GRÜTZMACHER, A.D. et al. Efeito de inseticidas e de tecnologia de aplicação no controle da lagarta-do-cartucho na cultura do milho no agroecossistema de várzea. In: REUNIÃO TÉCNICA ANUAL DO MILHO, 45.; REUNIÃO TÉCNICA ANUAL DO SORGO, 28., 2000, Pelotas, RS. Anais... Pelotas: Embrapa-CРACT, 2000a. p.567-573. (Embrapa-CPACT. Documentos, 70).

GRÜTZMACHER, A.D. et al. Insetos-pragas das culturas do milho e do sorgo no agroecossistema de várzea. In: PARFITT, J.M.B. (Coord. Produção de milho e sorgo em várzea. Pelotas: Embra). Clima Temperado, 2000b. p.87-101. (Embrapa Clima Temperado. Documentos, 74).

MARTINEZ, S.S. O Nim - Azadirachta indica - natureza, usos múltiplos, produção. Londrina: IAPAR, 2002. 142p.

MURUA, M.G.; VIRLA, E.G. Presencia invernal de Spodoptera frugiperda (Smith) (Lepidoptera: Noctuidae) en el área maicera de la Provincia de Tucumán, Argentina. Revista de la Facultad de Agronomia, La Plata, v.105, n.2, p.46-52, 2004.

PÉREZ, G. et al. Efecto de tres plaguicidas naturales derivados del nim sobre el combate de plagas en col y maíz en el campo, y en Vigna unguiculata en almacenamiento. Agronomía Costarricense, Cienfuegos, v. 21, n.2, p.259-266, 1997.

PORTO, M.P. et al. Milho em várzeas de clima temperado na região sul do Brasil: cultivares e manejo de solo e água. Pelotas: Embrapa Clima Temperado, 1998. 31p. (Embrapa Clima Temperado: Circular Técnica, 6).

R DEVELOPMENT CORE TEAM 2006. R: A language and environment for statistical computing. Vienna, Austria: R Foundation for Statistical Computing, ISBN 3-900051-07-0, Capturado em 15 jan. 2007. Online. Disponível na Internet: http://www.R-project.org

SCHMUTTERER, H. Properties and potential of natural pesticides from the neem tree, Azadirachta indica. Annual Review of Entomology, Palo Alto, v.35, p.271-297, 1990.

SILVA, M.T.B. Fatores que afetam a eficiência de inseticidas sobre Spodoptera frugiperda Smith em milho. Ciência Rural, Santa Maria, v.29, n.3, p.383-387, 1999

SIMMONDS, M.S.J. Molecular- and chemo-systematics: do they have a role in agrochemical discovery? Crop Protection, Oxford, v.19, n.8, p.591-596, 2000.

SOSA, M.A. Estimación del daño de Spodoptera frugiperda Smith (Lepidoptera: Noctuidae) en maíz con infestación natural en tres fechas de siembra en el noroeste santafesino. Santa Fe: INTA. EEA Reconquista, 2002. p.45. (Información para Extensión, 70).

VENDRAMIM, J.D. Uso de plantas inseticidas no controle de pragas. In: CICLO DE PALESTRAS SOBRE AGRICULTURA ORGÂNICA, 2., 1997, São Paulo. Palestras... Campinas: Fundação Cargill, 1997. p.64-69.

VENDRAMIM, J.D.; CASTIGLIONI, E. Aleloquímicos, resistência de plantas e plantas inseticidas. In: GUEDES, C. et al. (Org.). Bases e técnicas do manejo de insetos. Santa Maria: UFSM/CCR/DFS, 2000. p.113-128.

WAQUIL, J.M. et al. Controle da lagarta-do-cartucho em milho com inseticidas químicos e biológicos. Pesquisa Agropecuária Brasileira, Brasília, v.17, n.2, p.163-166, 1982.

WILLIAMS, W.P.; DAVIS, F.M. Response of corn to artificial infestation with fall armyworm and southwestern corn borer larvae. Southwestern Entomologist, Dallas, v.15, p.163166, 1990 